\title{
Clinical Significance of Atypical Squamous Cells of Undetermined Significance in Detecting Preinvasive Cervical Lesions in Post- Menopausal Turkish Women
}

\author{
Aytekin Tokmak ${ }^{1 *}$, Ali Irfan Guzel ${ }^{1}$, Emre Ozgu ${ }^{1}$, Murat $\mathbf{O z}^{1}$, Serap Akbay², \\ Salim Erkaya ${ }^{1}$, Tayfun Gungor ${ }^{1}$
}

\begin{abstract}
Background: To evaluate the clinical significance of atypical squamous cells of undetermined significance (ASCUS) in PAP test in post-menopausal women and compare with reproductive age women. Materials and Methods: A total of 367 patients who referred to our gynecologic oncology clinic were included to the study between September 2012 and August 2013. Data for 164 post-menopausal (group 1) and 203 pre-menopausal (group 2) women with ASCUS cytology were evaluated retrospectively. Immediate colposcopy and endocervical curettage was performed for both groups and conization for all women with a result suggestive of CIN2-3. Histopathological results and demographic features of patients were compared between the two groups. Results: Mean age of the patients was 54.6 \pm 6.5 years in group 1 and 38 \pm 6.6 years in group 2. Some $14(8.5 \%)$ of postmenopausal women and $36(17.7 \%)$ of pre-menopausal women were current smokers $(p=011)$. Totals of 38 $(23.2 \%)$ post-menopausal and $64(31.5 \%)$ pre-menopausal women were assessed for HPV-DNA. High risk HPV was detected in $7(4.3 \%)$ and $21(10.3 \%)$, respectively $(p=0.029)$. Final histopathological results recorded were normal cervix, low grade cervical intra-epithelial neoplasia (CIN 1), and high grade cervical intra-epithelial neoplasia (CIN2-3). In group 1 results were $84.8 \%, 12.2 \%$ and $1.8 \%$, respectively, and in group 2 were $71.9 \%$, 23.2\% and 4.9\%. There were no cases of micro invasive or invasive cervical carcinoma in either group. Two cases were detected as endometrial carcinoma in the menopausal group (1.2\%). Conclusions: In current study we found that preinvasive lesions were statistically significantly higher in pre-menopausal women than postmenopausal women with ASCUS. Cervicitis was more common in menopausal women. Therefore, we think that in case of ASCUS in a post-menopausal woman there is no need for radical management.
\end{abstract}

Keywords: ASCUS - post-menopausal women - reproductive age women - preinvasive lesions

Asian Pac J Cancer Prev, 15 (16), 6639-6641

\section{Introduction}

Worldwide, cervical cancer is the third most common cancer among women and the second most frequent cause of cancer-related death, accounting for nearly 300,000 deaths annually (Filipi and Xhani, 2014). In developing countries cervical cancer is a common problem with an incidence of $15 \%$ of female cancers with a risk of $1.5 \%$ at age below 65 years old (Parkin and Bray, 2006). Papanicolaou smear is a cheap, simple and commonly used technique for screening cervical cancer and preinvasive cervical lesions. Pap smear and regular cervical cancer screening programs reduced the mortality rate due to cervical cancer and allowed detection and treatment of preinvasive cervical lesions (Siegel et al., 2012; Cakmak et al., 2014). ASCUS was first described by the Bethesda System (TBS) in 1988 with prevalence in general population of 3 to $10 \%$ (Kurman et al., 1994; Solomon et al., 2002). Management of ASCUS includes; repeat cytology, colposcopy and/or HPV DNA testing (Poomtavorn et al., 2011). In pre-menopausal and post-menopausal age women atrophic changes due to hypoestrogenism often diagnosed as ASCUS and resulting in over diagnosis and over treatment (Rodriguez et al., 2012).

In this study, we aimed to evaluate the clinical significance of atypical squamous cells of undetermined significance in detecting preinvasive cervical lesions in post-menopausal women and compare those with reproductive age women.

\section{Materials and Methods}

This retrospective case control study was conducted in Dr. Zekai Tahir Burak Women's Health Education and Research Hospital, Ankara, Turkey; between September 2012 and August 2013. This is a tertiary research hospital in the middle region of the country. Most of the health 
services are provided by the government and free of charge. The study was approved by the Ethics Committee of Dr. Zekai Tahir Burak Women's Health Research and Education Hospital. Due to the retrospective design informed content was not taken.

A total of 367 patients 164 post-menopausal (group 1) and 203 pre-menopausal (group 2) women who underwent Pap smear for screening purpose and resulted in with ASCUS cytology was included to the study. Women on hormonal replacement therapy (HRT), who were pregnant and/or had been previously treated for CIN were excluded from the study. Pap smear was performed by using conventional cytobrush technique and evaluated according to the 2001 Bethesda. Cervical brush specimens were collected in Cell Collection Media System (Cobas ${ }^{\circledR}$ PCR, Roche Diagnostic GmbH, IN, USA) for HPV DNA testing. DNA was purified with the Magna Pure DNA isolation kit I on the MagNa Pure LC System (Roche Diagnostics, IN, USA). After nucleic acid isolation, all samples were analyzed by Roche Linear Array HPV Genotyping Test (Roche Diagnostics, IN, USA). HPV DNA was assessed using polymerase chain reaction (PCR) to amplify the viral DNA, followed by detecting the presence and types of HPV nucleic acid hybridization using a reverse hybridization system (High risk types were HPV-16, 18, $31,33,35,39,45,51,52,56,58,59,66,68,82)$.

All of the patients underwent immediate colposcopy and endocervical curettage and conization was performed to all women with a suggestive result for CIN2-3 by an experienced surgical team.

Data recorded were; age, gravidity, duration of menopause, smoking status, HPV DNA status, and post conization histopathological results. The association of these parameters was assessed between demographic and clinical features.

\section{Statistics}

Means and standard deviations (SD) were calculated for continuous variables. Subject characteristics and demographics were analyzed descriptively. The normal distribution of the variables was analyzed by the Kolmogorov Smirnov test. The Student's t-test and chisquare test was used to evaluate associations between the categorical and continuous variables. All variables were included in the backward stepwise procedure. Twosided P-values were considered statistically significant at $p<0.05$. Statistical analyses were carried out using the statistical package SPSS 15.0 for Windows (SPSS Inc., Chicago, IL, USA).

\section{Results}

A total 367 patients with the cytological diagnosis of ASCUS were included in the study. One hundred and sixty four post-menopausal women and two hundred and three pre-menopausal women were compared in this study. Mean age of the patients was 54.6 \pm 6.5 years in group1 and $38 \pm 6.6$ years in group 2. Mean duration of menopause was $6.5 \pm 5.8(1-24)$ years in group $1.14(8.5 \%)$ of postmenopausal women and $36(17.7 \%)$ of pre-menopausal women were current smokers $(\mathrm{p}=0.11)$. Satisfactory
Table 1. Demographic and Clinical Features of the Patients

\begin{tabular}{lccc}
\hline & $\begin{array}{c}\text { Postmenopausal } \\
\text { age women (n:164) }\end{array}$ & $\begin{array}{c}\text { Premenopausal } \\
\text { age women (n:203) }\end{array}$ & $\mathrm{p}$ \\
\hline Age (years) & $54.6 \pm 6.5$ & $38.0 \pm 6.6$ & $<0.001$ \\
Gravida & $3.4 \pm 1.6$ & $2.1 \pm 1.0$ & $<0.001$ \\
Parity & $2.6 \pm 1.2$ & $1.7 \pm 0.8$ & $<0.001$ \\
Abortus & $0.7 \pm 0.8$ & $0.3 \pm 0.5$ & $<0.001$ \\
Smoking & $8.5(14)$ & $17.7(36)$ & 0.011 \\
Adequacy of smear & $95.7(157)$ & $96.1(195)$ & 0.81 \\
Satisfactory colposcopy & $68.2(112)$ & $84.7(172)$ & $<0.001$ \\
HPV DNA test & & & \\
Available & $23.2(38)$ & $31.5(64)$ & 0.076 \\
Positive & $4.3(7)$ & $10.3(21)$ & 0.029 \\
Negative & $95.7(157)$ & $89.7(182)$ & 0.088 \\
Preinvasive lesion & $15.2(\mathrm{n}: 25)$ & $28.1(\mathrm{n}: 57)$ & 0.003 \\
\hline *Data are presented as mean \pm SD and \%(n). P<0,05 is considered statistically significant
\end{tabular}

Table 2. Final Histopathologic Results for the Patients

\begin{tabular}{lccc}
\hline Final diagnosis & $\begin{array}{c}\text { Postmenopausal } \\
\%(\mathrm{n})\end{array}$ & $\begin{array}{c}\text { Premenopausal } \\
\%(\mathrm{n})\end{array}$ & $\mathrm{p}$ \\
\hline Normal & $55.5(91)$ & $55.7(113)$ & 0.001 \\
Cervicitis & $29.3(48)$ & $16.3(33)$ & \\
CIN1 & $12.2(20)$ & $23.2(47)$ & \\
CIN2-3 & $1.8(3)$ & $4.9(10)$ & \\
Microinvasive cancer & 0 & 0 & \\
\hline
\end{tabular}

*Data presented as $\mathrm{n}(\%)$

colposcopic examination was significantly higher in pre-menopausal women $(84.7 \%$ vs $68.2 \%)$. Smear adequacy was $95.7 \%$ and $96.1 \%$ of group 1 and group 2; respectively $(\mathrm{p}=0.81) .38(23.2 \%)$ post-menopausal and $64(31.5 \%)$ pre-menopausal women were assessed for HPV-DNA. High risk HPV was detected seven (4.3\%) of them and $21(10.3 \%)$ of them in group 1 and group 2 ; respectively $(\mathrm{p}=0.029)$. Demographical and clinical features were summarized in Table 1 . All of the patients underwent immediate colposcopy and colposcopy guided cervical biopsy and endocervical curettage was performed. Biopsy and endocervical curettage results were available for each woman (Table 2). The most common findings were normal cervix in both groups (55.5\% vs $55.7 \%$ ). Cervicitis was more common in post-menopausal group (29.3\% vs $16.3 \%)$. Women with a suggestive result for CIN2-3 underwent conization. There were no cases of micro invasive or invasive cervical carcinoma in either group. Two cases were detected as endometrial carcinoma in menopausal group (1.2\%).

\section{Discussion}

In current study, we compared the demographic and clinical features of atypical squamous cells of undetermined significance (ASCUS) in PAP test in postmenopausal women and compare with reproductive age women. A total of 164 post-menopausal (group 1) and 203 pre-menopausal (group 2) women with ASCUS cytology was included to the study. We found statistically significant differences between the groups in terms of demographic and obstetric history. Smoking was more common in premenopausal women. Colposcopy was more satisfactory in pre-menopausal age women. The high risk HPV virus positivity was statistically significantly higher in premenopausal group. We also detected significantly more 
preinvasive lesions in pre-menopausal age women.

ASCUS is defined as presence of abnormal but not precancerous squamous cells in the Pap smear (Pity et al., 2012). The management of these pathology includes immediate colposcopy with or without biopsy, HPV testing and follow up with subsequent Pap smears in 4-6 months (Poomtavorn et al., 2011). Solomon et al. (2001) reported that reported that follow up with 4-6 months of Pap smear has a sensitivity of $85 \%$ and reduces the cost of unnecessary colposcopy but also may result in delay in diagnosis of preinvasive cervical lesions. American Society for Colposcopy and Cervical Pathology (ASCCP) recommended that in ASCUS patients HPV DNA testing with liquid-based testing technique should be the first line strategy (Wright et al., 2002) and Sangkarat et al. (2014) reported their experience on Siriraj liquid-based cytology. In this study, we also determined HPV status of the patients but we did not have liquid-based testing material and this is the limitation of our study.

Previous studies reported that HPV DNA has a favor of decline as the age of the women increase (Johnstone and Logani, 2007). Castle et al. (2005) reported that in cervical HPV DNA positive women with newly apparent infections decreased, whereas persistence increased with age. In a previous study authors found that with increasing pathological grade, the positive rate of high risk HPV also increased (Wang et al., 2013). We also found similar results to these studies. In our post-menopausal group women HPV DNA positivity and preinvasive lesions were lower than the premenopausal age women. Kececioglu et al. (2013) reported that HPV DNA testing results in more cases with high grade preinvasive lesions than immediate colposcopy in women who had ASCUS on first PAP test. But, while this approach reduces the referral rate to colposcopy, it could increase the cost of management.

In post-menopausal women, hormonal changes especially hypoestrogenism that results in vaginal and cervical atrophy is a risk factor for Pap smear abnormalities (Cakmak et al., 2014). Piccoli et al. (2008) evaluated 254 post-menopausal women with atypical squamous cells and low squamous intraepithelial lesions in Pap smear; they recommended a single course of local estrogen replacement therapy in these women in order to prevent unnecessary treatments.

Goksedef et al. (2013) also designed a study and evaluated the clinical characteristics of post-menopausal women with ASCUS and proposed that hypoestrogenism should be treated before invasive procedures in these women. They also found that these women had more benign histological changes than pre-menopausal women. They also recommend managing post-menopausal women with ASCUS in the same manner as pre-menopausal women.

We had several limitations in our study including; retrospective design, conventional cytobrush technique for Pap smear, lack of follow up, biopsies in inadequate smear and/or unsatisfactory colposcopy and no treatment to atrophy.

In conclusion, we think that management of postmenopausal women with ASCUS should be similar to those in pre-menopausal age women. These strategies prevent over diagnosis and over management.

\section{References}

Cakmak B, Koseoglu DR (2014). Comparison of cervical cytological screening results between post-menopausal and elderly women. Turk Patoloji Derg, 30, 38-42.

Castle PE, Schiffman M, Herrero R, et al (2005). A prospective study of age trends in cervical human papillomavirus acquisition and persistence in Guanacaste, Costa Rica. $J$ Infect Dis, 191, 1808-16.

Filipi K, Xhani A (2014). Assessment of cervical cytological data in Albanian females. Asian Pac J Cancer Prev, 15, 2129-32.

Goksedef BP, Akbayir O, Baran SY, et al (2011). Atypical squamous cells of undetermined significance in postmenopausal women: a comparative retrospective analysis. Eur J Obstet Gynecol Reprod Biol, 159, 418-21.

Johnston EI, Logani S (2007). Cytologic diagnosis of atypical squamous cells of undetermined significance in perimenopausal and post-menopausalwomen. Lessons leamed from human papilloma virus DNA testing. Cancer, 111, 160-5.

Kececioglu M, Seckin B, Baser E, et al (2013). Cost and effectiveness comparison of immediate colposcopy versus human papillomavirus DNA testing in management of atypical squamous cells of undetermined significance in Turkish women. Asian Pac J Cancer Prev, 14, 511-4.

Kurman R, Henson D, Herbst A, et al (1994). Interim guidelines for management of abnormal cervical cytology. JAMA, 27, 1866-9.

Parkin DM, Bray F (2006). Chapter 2: The burden of HPVrelated cancers. Vaccine, 24, 3-11.

Piccoli R, Mandato VD, Lavitola G, et al (2008). Atypical squamous cells and low squamous intraepithelial lesions in post-menopausal women: implications for management. Eur J Obstet Gynecol Reprod Biol, 140, 269-74.

Poomtavorn Y, Suwannarurk K, Thaweekul Y, Maireang K. (2011). Risk factors for high-grade cervical intraepithelial neoplasia in patients with atypical squamous cells of undetermined significance (ASC-US) Papanicolaou smears. Asian Pac J Cancer Prev, 12, 235-8.

Rodriguez EF, Reynolds JP, Jenkins SM, et al (2012). Atypical squamous cells of undetermined significance in patients with HPV positive DNA testing and correlation with disease progression by age group - an institutional experience. Int $J$ Clin Exp Pathol, 5, 428-35.

Sangkarat S, Laiwejpithaya S, Rattanachaiyanont M, et al (2014). Performance of Siriraj liquid-based cytology: a single center report concerning over 100,000 samples. Asian Pac J Cancer Prev, 15, 2051-5.

Siegel R, Naishadham D, Jemal A (2012). Cancer statistics, 2012. CA Cancer J Clin, 62, 10-29.

Solomon D, Schiffman M, Tarone R, ALTS Study Group (2001). Comparison of three management strategies for patients with atypical squamous cells of undetermined significance: baseline results from a randomized trial. J Natl Cancer Inst, 93, 293-9.

Solomon D, Davey D, Kurman R, et al (2002). The 2001 Bethesda System: terminology for reporting results of cervical cytology. JAMA, 287, 2114-9.

Wang JL, Yang YZ, Dong WW, et al (2013). Application of human papillomavirus in screening for cervical cancer and precancerous lesions. Asian Pac J Cancer Prev, 14, 2979-82.

Wright TC Jr, Cox JT, Massad LS, Twiggs LB, Wilkinson EJ. (2002). 2001 Consensus Guidelines for the management of women with cervical cytological abnormalities. JAMA, 287, 2120-9. 